EGU21-15603, updated on 18 Jan 2022

https://doi.org/10.5194/egusphere-egu21-15603

EGU General Assembly 2021

(c) Author(s) 2022. This work is distributed under

the Creative Commons Attribution 4.0 License.

\title{
EnVAR for ICON-LAM: observations and quality control
}

\author{
Mareike Burba ${ }^{1}$, Sven Ulbrich ${ }^{1}$, Stefanie Hollborn ${ }^{1}$, Roland Potthast ${ }^{1}$, and Peter Knippertz ${ }^{2}$ \\ ${ }^{1}$ Deutscher Wetterdienst, Forschung und Entwicklung, Offenbach, Germany (mareike.burba@dwd.de) \\ ${ }^{2}$ Karlsruhe Institute of Technology, Karlsruhe, Germany
}

The German Weather Service (DWD) introduces the regional NWP model ICON-LAM (ICON Limited Area Mode) in 2021 to replace the COSMO model. For the ICON-LAM data assimilation, a novel EnVAR (Ensemble VARiational data assimilation) setup is currently evaluated in comparison to the operational deterministic run of KENDA-LETKF (Local Ensemble Transform Kalman Filter). This requires special care as the observation handling differs for the global assimilation (via EnVAR) and the regional assimilation (KENDA). Furthermore, the variational quality control for the regional EnVAR may require a setup differing from the global setup. We will give an introduction to the observation processing in DWD's data assimilation framework (DACE).

For future development, we give an outlook on how a regional EnVAR can be used for a regional deterministic analysis by using a global ICON ensemble in combination with a regional deterministic ICON-LAM run. This is potentially of interest for DWD's partners with smaller computational capacities, because a regional EnVAR analysis is computationally less expensive than running a full KENDA ensemble assimilation cycle. 